\section{(C) OPEN ACCESS}

\begin{abstract}
- Additional material is published online only. To view please visit the journal online (http://dx.doi.org/10.1136/ bjsports-2016-097089).

${ }^{1}$ Sports Medicine Research Department, Sports Surgery Clinic, Dublin, Ireland ${ }^{2}$ Department of Life Sciences, University of Roehampton, Roehampton, UK

${ }^{3}$ Centre for Health, Exercise and Sports Medicine, University of Melbourne, Melbourne, Victoria, Australia

${ }^{4}$ School of Health and Human Performance, Dublin City University, Dublin, Ireland ${ }^{5}$ Insight Research Centre, Dublin City University, Dublin, Ireland ${ }^{6}$ Department of Medicine, University College Cork, Cork, Ireland
\end{abstract}

Correspondence to Mr Enda King, Sports Medicine Research Department, Sports Surgery Clinic, Santry Demesne, Dublin 9, Republic of Ireland; endaking@hotmail.com

Accepted 31 December 2017 Published Online First 17 March 2018

\title{
Clinical and biomechanical outcomes of rehabilitation targeting intersegmental control in athletic groin pain: prospective cohort of 205 patients
}

\author{
Enda King, ${ }^{1,2}$ Andrew Franklyn-Miller, ${ }^{1,3}$ Chris Richter, ${ }^{1}$ Eamon O'Reilly, ${ }^{1}$ Mark Doolan, ${ }^{1}$ \\ Kieran Moran, ${ }^{4,5}$ Siobhan Strike, ${ }^{2}$ Éanna Falvey ${ }^{1,6}$
}

\begin{abstract}
Background Clinical assessments and rehabilitation in athletic groin pain (AGP) have focused on specific anatomical structures and uniplanar impairments rather than whole body movement.

Objective To examine the effectiveness of rehabilitation that targeted intersegmental control in patients with AGP and to investigate post rehabilitation changes in cutting biomechanics.
\end{abstract}

Methods Two hundred and five patients with AGP were rehabilitated focusing on clinical assessment of intersegmental control, linear running and change of direction mechanics in this prospective case series. Hip and Groin Outcome Score (HAGOS) was the primary outcome measure. Secondary measures included painfree return to play rates and times, pain provocation on squeeze tests and three-dimensional (3D) biomechanical analysis during a $110^{\circ}$ cutting manoeuvre.

Results Following rehabilitation, patients demonstrated clinically relevant improvements in HAGOS scores (effect size (ES): 0.6-1.7). 73\% of patients returned to play pain-free at a mean of 9.9 weeks ( \pm 3.5 ). Squeeze test values also improved (ES: 0.49-0.68). Repeat 3D analysis of the cutting movement demonstrated reductions in ipsilateral trunk side flexion (ES: 0.79) and increased pelvic rotation in the direction of travel (ES: 0.76). Changes to variables associated with improved cutting performance: greater centre of mass translation in the direction of travel relative to centre of pressure (ES: 0.4 ), reduced knee flexion angle (ES: 0.3 ) and increased ankle plantar flexor moment (ES: 0.48) were also noted.

Conclusions Rehabilitation focused on intersegmental control was associated with improved HAGOS scores, high rates of pain-free return to sporting participation and biomechanical changes associated with improved cutting performance across a range of anatomical diagnoses seen in AGP.

\section{INTRODUCTION}

Athletes with athletic groin pain (AGP) frequently present with single or multiple painful anatomical sites in the groin. ${ }^{12}$ Coexisting painful structures along with a lack of histological pathology studies ${ }^{3}$ make it challenging to identify a specific diagnosis on which to focus treatment. ${ }^{1}$ Attempts have been made to group painful structures by both anatomy ${ }^{4}$ and entity, ${ }^{2}$ but the descriptive terminology in use remains both wide and confusing, ${ }^{5}$ and there have been recent attempts to simplify using expert consensus. ${ }^{6}$
No clear differences in outcomes on return to play (RTP) times or rates, between surgical intervention and rehabilitation, were found in a recent systematic review. ${ }^{7}$ The success of exercise-based rehabilitation for AGP has been documented. ${ }^{8} 9$ Programmes targeting lumbopelvic control and muscle strength, focusing on adductor strengthening, have been compared with manual therapy ${ }^{10}$ and with rest and active recovery ${ }^{11}$ with good outcomes at long-term follow-up. ${ }^{12}$ These studies commonly included patients with a single anatomical presentation: adductor-related groin pain. This limits their generalisability in treating athletes presenting with other entities. ${ }^{71011}$

Outcome measures used to assess interventions in AGP include patient-reported outcome measures such as the Hip and Groin Outcome Score (HAGOS), and strength and pain provocation measures such as adductor squeeze testing. ${ }^{13} 14$ To date, despite multiplanar movement patterns (such as change of direction and high-speed sprinting) being reported as provocative activities in athletes with AGP, ${ }^{15-18}$ these have not been used as outcome measures. The physical demands of acceleration and braking (common in field sports) are not evenly distributed across the hip, knee and ankle but depend heavily on the relationship between the centre of mass and centre of pressure. ${ }^{19} 20$ This complex relationship is not controlled by a single muscle group that can be targeted in isolation.

Three-dimensional (3D) motion analysis enables the evaluation of complex multiplanar, multijoint movements. ${ }^{21}{ }^{22}$ While performing a cutting manoeuvre, patients with AGP could be characterised into three distinct movement strategies (clusters). These biomechanical clusters did not correlate with a specific site of groin pain. ${ }^{23}$ An individual athlete's risk of developing AGP is likely linked to a combination of individual risk factors such as training load, recovery and sport/position. ${ }^{24-26} \mathrm{We}$ hypothesise that an athlete's movement strategy may also be part of this equation, leading to a biomechanical overload resulting in symptoms. As a result, an intervention focusing on intersegmental control is a novel approach to rehabilitation in AGP.

The aim of this study was to describe the outcome of a rehabilitation programme focusing on intersegmental control, in consecutive patients who presented with a variety of anatomical diagnoses relating to AGP. The primary outcome measure used was the HAGOS questionnaire. Secondary measures included RTP 


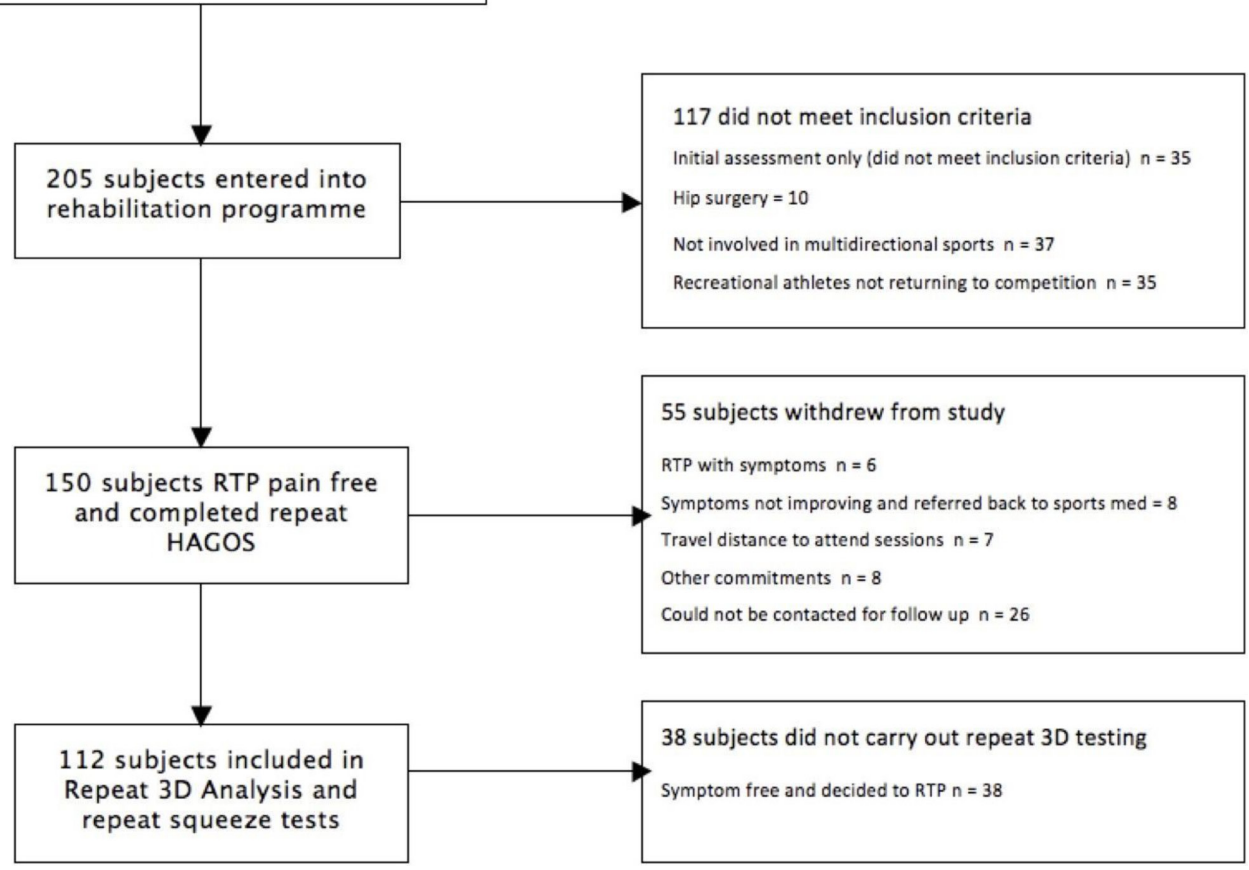

Figure 1 Flow chart of patient inclusion in study. 3D, three-dimensional; HAGOS, Hip and Groin Outcome Score; RTP, return to play.

rates and times, pain provocation tests (squeeze test) and 3D biomechanical analysis of cutting. It was hypothesised that the rehabilitation intervention would be effective across all clinical outcomes irrespective of anatomical diagnosis.

\section{METHODS}

Three hundred and twenty-two male patients who presented to the sports medicine department of Sports Surgery Clinic, Dublin, from January 2013 to May 2015 were assessed for eligibility in this study. Patients presented to the clinic independently or by third-party referral. Of the 322 patients, 205 were eligible for inclusion in the study (figure 1). All patients signed informed consent. The methodology of the study reported is in keeping with the Strengthening the Reporting of Observational Studies in Epidemiology guidelines. ${ }^{27}$

\section{Diagnostic assessment}

A sports and exercise medicine physician performed an injury history (including sport participation and duration of symptoms) and clinical examination, which included initial interpretation of MRI to make an anatomical diagnosis and exclude pathology unsuitable for rehabilitation. The clinical assessment, radiological findings and differential diagnostic process have been detailed elsewhere. ${ }^{1}$

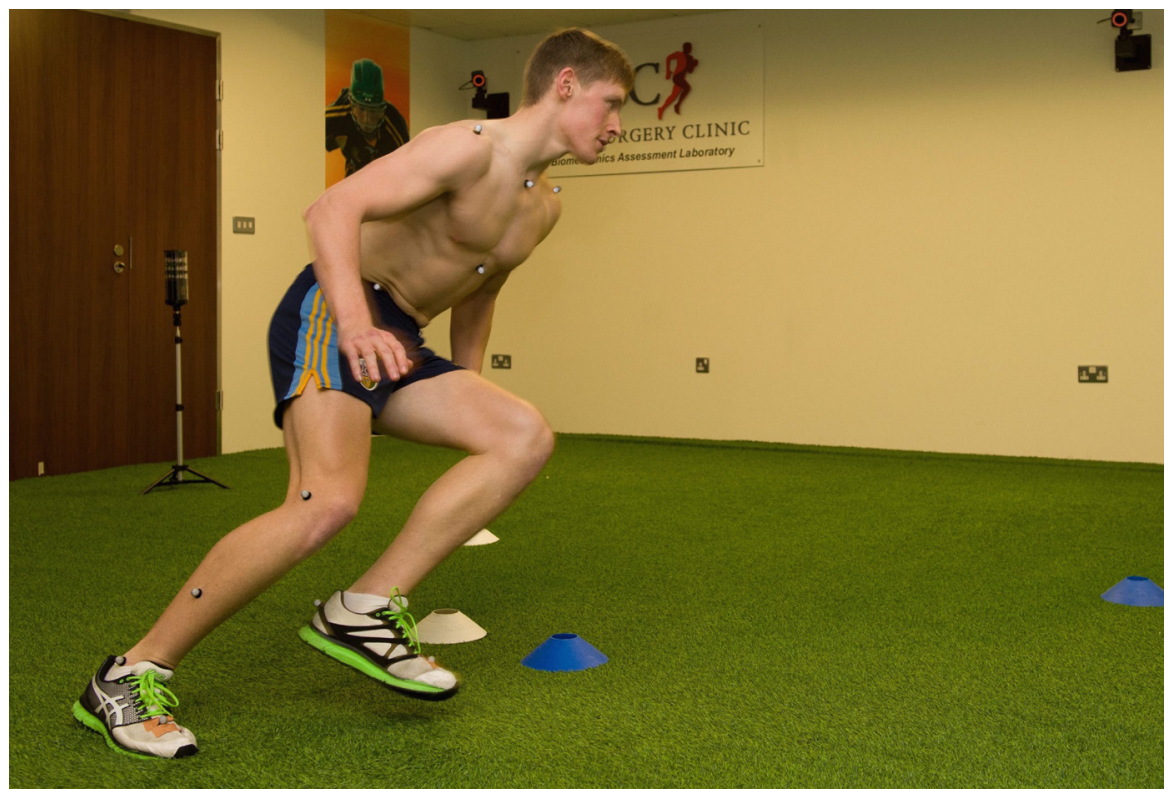

Figure $2110^{\circ}$ change of direction test. 
SAGITTAL

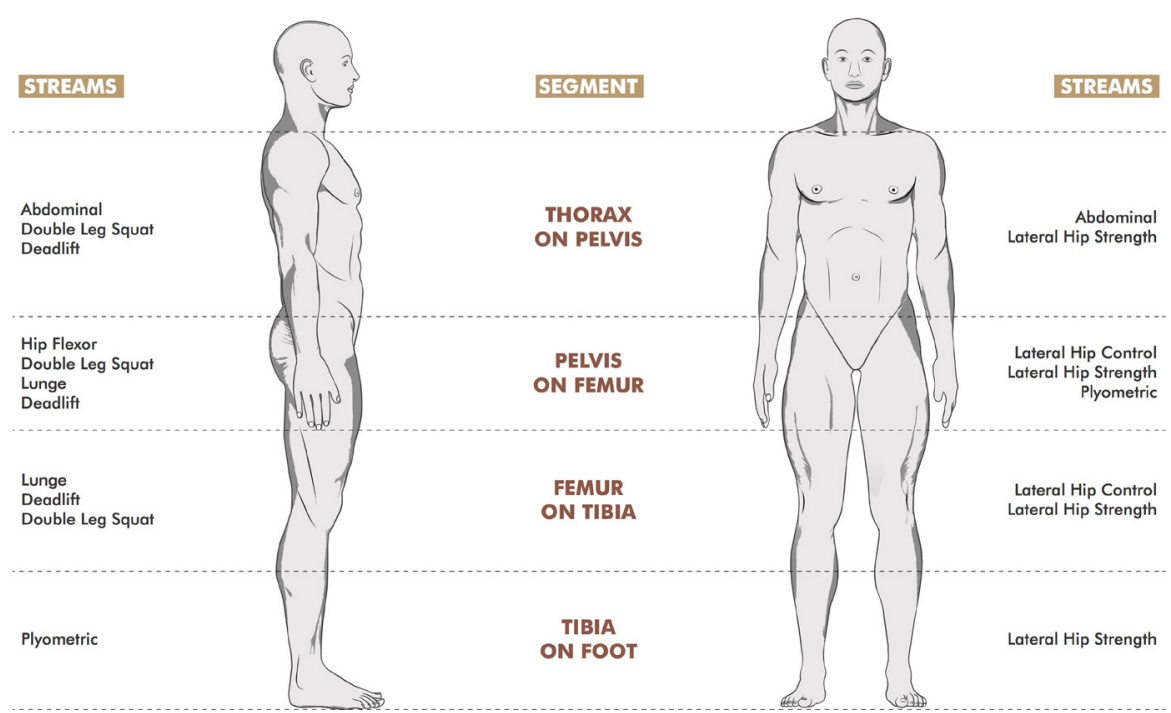

Figure 3 Level 1: intersegmental control and strength rehabilitation streams (the figure identifies the plane of intersegmental control each stream influenced).

\section{Inclusion criteria}

All patients reported pain in the anterior hip and groin area during their chosen sporting activity, and symptom duration was greater than 4 weeks. All patients had a stated intention of returning to the same level of preinjury participation in competitive multidirectional sport. All patients with an anatomical diagnosis falling under AGP (iliopsoas, adductor, pubic aponeurosis and hip) were included as per Falvey et al. ${ }^{1}$

\section{Exclusion criteria}

Patients with hip joint arthrosis (grade 3 or higher on $\mathrm{MRI}^{28}$ ); those who did not intend to return to preinjury activity levels; those who could not commit to completing the rehabilitation programme as prescribed due to time or equipment/facility constraints; and those with underlying medical conditions such as inflammatory arthropathy or infection were excluded.
Patients eligible for inclusion completed the HAGOS questionnaire as the primary outcome measure on initial assessment and on discharge to RTP, which has been shown to be a reliable measure (Intraclass Correlation: 0.82-0.91), with a smallest detectable change at group level of 2-7-5.2 and an SE of measure of 6.4-12.2. ${ }^{29}$

\section{Clinical assessment}

Those patients eligible for inclusion underwent a physical assessment with a senior physiotherapist documenting pain provocation tests using crossover test ${ }^{17}$ and squeeze tests at $0^{\circ}, 45^{\circ}$ and $90^{\circ},{ }^{30}$ and the symmetry of hip internal rotation at $90^{\circ}$ hip flexion with a goniometer (Saehan). The pressure $(\mathrm{mm} \mathrm{Hg})$ at onset of the patient's groin pain and maximum pressure achievable were recorded using a sphygmomanometer (DS66, Welch Allyn), which was set at a pretest pressure of $20 \mathrm{~mm} \mathrm{Hg}$. The reliability of the squeeze test has been shown previously (ICC: 0.89-0.92) with an

\section{SAGITTAL}

FRONTAL / TRANSVERSE

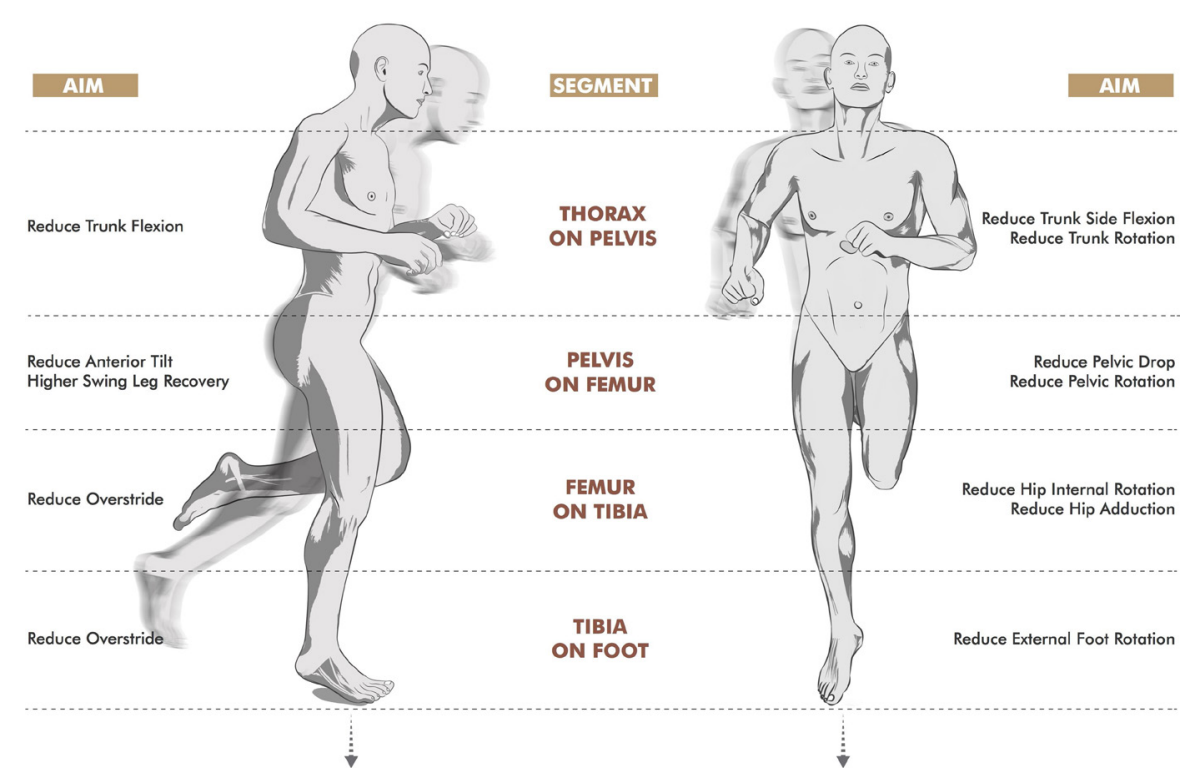

Figure 4 Level 2: segmental control focus of linear running drills (the figure demonstrates the intersegmental control the drills targeted). 


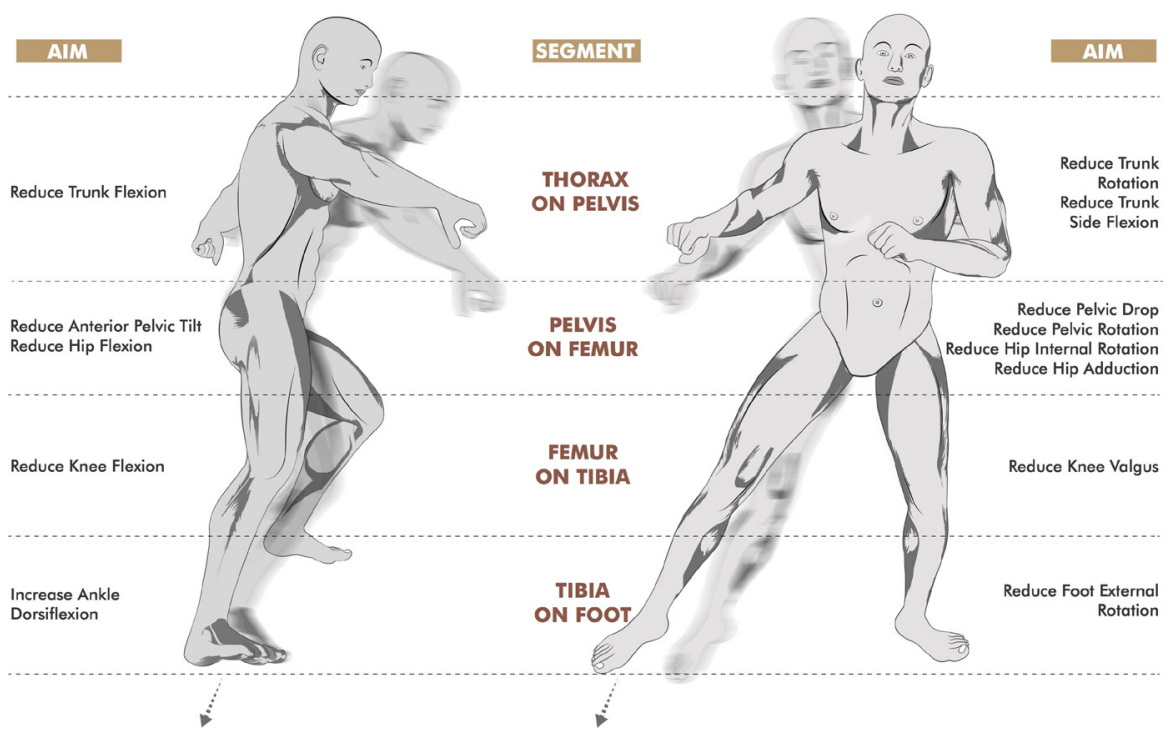

Figure 5 Intersegmental control focus of multidirectional drills (the figure demonstrates the intersegmental control the drills targeted).

SE of measurement between $1.6 \%$ and $3.3 \%,{ }^{30}$ and these tests have been reported as valid for use in clinical research on groin pain. ${ }^{31}$

\section{Biomechanical assessment}

Subsequently patients underwent 3D biomechanical analysis of a $110^{\circ}$ cut (figure 2 ). The cutting task was performed at the patient's perceived maximal effort for each trial. The approach speeds are reported to demonstrate comparability. The reliability (ICC >0.85), validity and methodology of this test have been reported previously. ${ }^{21} 232$ The biomechanical variables included in the analysis were joint angles and internal joint moments, joint powers (rate at which joint work is done) and joint work (change in angle multiplied by change in moment) for all three anatomical planes. Details regarding the method of calculation of these variables have been described previously. ${ }^{23}$

\section{Intervention}

The rehabilitation programme consisted of three levels. Level 1 addressed intersegmental control and strength (figure 3), level 2 involved linear running mechanics and increasing linear running load tolerance (linear A) (figure 4), and level 3 targeted multidirectional mechanics and the transition back to high intensity sprinting (linear B) (figure 5). The rehabilitation intervention is reported in detail in accordance with the Template for

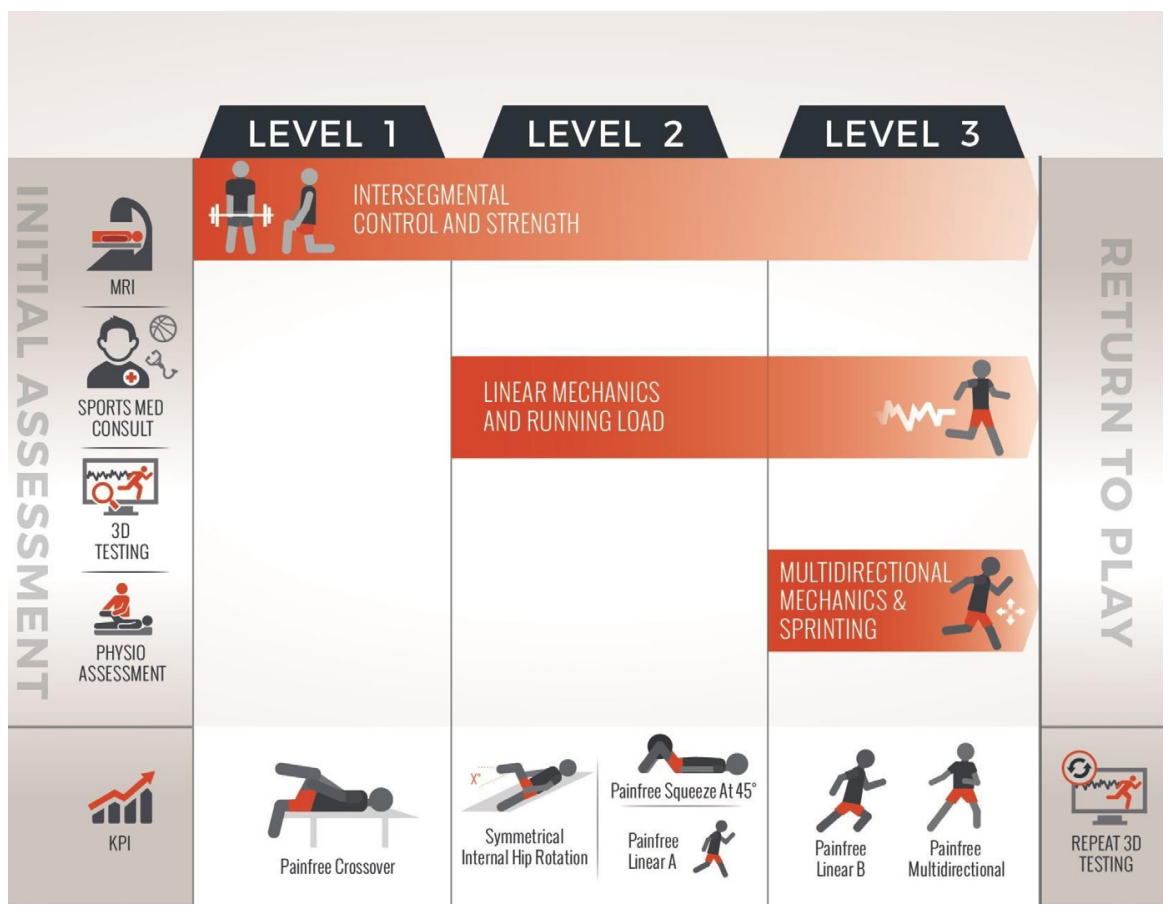

Figure 6 Components of rehabilitation and key performance indicators for progression. 


\begin{tabular}{|c|c|c|}
\hline & Time (IQR) & Range \\
\hline \multirow[t]{2}{*}{ Duration of symptoms } & 32 weeks (20-52) & 4-416 weeks \\
\hline & Total & Percentage \\
\hline \multicolumn{3}{|l|}{ Diagnosis } \\
\hline Pubic aponeurosis & 132 & 64 \\
\hline Iliopsoas & 8 & 4 \\
\hline Adductor & 35 & 17 \\
\hline Hip & 30 & 15 \\
\hline \multicolumn{3}{|l|}{ Side } \\
\hline Left & 75 & 37 \\
\hline Right & 88 & 43 \\
\hline Bilateral & 42 & 20 \\
\hline \multicolumn{3}{|l|}{ Sport } \\
\hline Gaelic Football & 131 & 64 \\
\hline Hurling & 29 & 14 \\
\hline Soccer & 25 & 12 \\
\hline Rugby & 15 & 7 \\
\hline Hockey & 5 & 2 \\
\hline \multicolumn{3}{|l|}{ 3D biomechanics } \\
\hline Cluster 1 & 88 & 43 \\
\hline Cluster 2 & 44 & 21 \\
\hline Cluster 3 & 74 & 36 \\
\hline
\end{tabular}

Intervention Description and Replication checklist and guide ${ }^{33}$ in online supplementary appendix A. The exercise selection was dictated by the patient's physical competency (ie. ability to perform the exercise with appropriate technique), and progression through the programme was individualised according to each patient achieving key goals for progression (figure 6).

Patients whose symptoms were not improving during rehabilitation in spite of improving segmental control were referred back to the sports medicine physician for review and were considered to have failed rehabilitation. Patients who withdrew were contacted regarding their reason for withdrawal.

Patients completed the HAGOS questionnaire after rehabilitation and pain-free RTP, and where possible pain provocation testing and $3 \mathrm{D}$ biomechanics were also repeated. Patients were cleared to RTP in their chosen sports once they had passed through the three rehabilitation levels and demonstrated symptom-free completion of linear $\mathrm{A}$ and $\mathrm{B}$ and multidirectional drills. Time to RTP was to their first full participation in training/ competition after being cleared. Where secondary testing revealed symptoms on squeeze testing or residual biomechanical asymmetries during cutting, in spite of pain-free completion of the running programme, further follow-up was performed. This was to ensure complete resolution of these deficits before discharge from the programme.
Table 3 Changes in squeeze test pre-rehabilitation and postrehabilitation $(n=112)(P<0.001)$

\begin{tabular}{|c|c|c|c|c|}
\hline \multicolumn{2}{|c|}{ Squeeze $(\mathrm{mm} \mathrm{Hg})$} & \multirow{2}{*}{$\frac{\text { Initial mean (SD) }}{81( \pm 28)}$} & \multirow{2}{*}{$\frac{\text { Discharge mean (SD) }}{\text { NA }}$} & \multirow[t]{2}{*}{ Effect size } \\
\hline $0^{\circ}$ & p1 & & & \\
\hline & r2 & $123( \pm 29)$ & $135( \pm 32)$ & 0.68 \\
\hline \multirow[t]{2}{*}{$45^{\circ}$} & p1 & $159( \pm 43)$ & NA & \\
\hline & r2 & $223( \pm 41)$ & $234( \pm 40)$ & 0.65 \\
\hline \multirow[t]{2}{*}{$90^{\circ}$} & p1 & $122( \pm 45)$ & NA & \\
\hline & r2 & $177( \pm 41)$ & $209( \pm 38)$ & 0.46 \\
\hline
\end{tabular}

$\mathrm{p} 1$ is pressure at initial onset of patients groin pain symptoms; $\mathrm{r} 2$ is maximum pressure achieved; $\mathrm{Na}$, not applicable.

\section{Statistical analysis}

Data analysis was carried out using SPSS V.21.0 to report descriptive statistics for patient data. Spearman's rank correlation was used to correlate RTP time and duration of symptoms prior to rehabilitation. One-way analysis of variance was used to calculate the difference in RTP time between anatomical diagnoses and movement clusters at initial assessment. Paired samples t-test was used to analyse the change in HAGOS and squeeze test scores after rehabilitation. To examine if differences exist between the kinematic and kinetic measures post rehabilitation, statistical parametric mapping (1D, paired t-test) was used. ${ }^{34}$ Effect sizes were calculated in a point-by-point manner, and reported according to Cohen's $\mathrm{d}(\mathrm{d}>0.3=$ small; $\mathrm{d}>0.5$ $=$ medium; $\mathrm{d}>0.7=$ strong). ${ }^{35}$ Data processing and statistical parametric mapping were performed using MATLAB (R2015a, MathWorks, Natick, MA, USA).

\section{RESULTS}

Two hundred and five patients entered rehabilitation $(24.9 \pm 5.1$ years; $179.4 \pm 5.8 \mathrm{~cm} ; 80.4 \pm 9.2 \mathrm{~kg}$ ), with a median duration of symptoms of 32 weeks (IQR: 20-52 weeks) and participated across a range of field sports (table 1 ). The most commonly identified anatomical diagnoses were pain or tenderness at the pubic aponeurosis (64\%), which was superior to the pubic symphysis, with $17 \%$ adductor, $15 \%$ hip and $4 \%$ iliopsoas, with $20 \%$ reporting bilateral symptoms.

Fifty-five patients (27\%) commenced the rehabilitation process but did not complete repeat Hip and Groin Outcome Score. Eight of these patients' symptoms failed to resolve during rehabilitation and were removed from the study (pubic aponeurosis $=5$; adductor $=2$; hip $=1$.) Reasons reported for dropout prior to discharge included desire to return to play in spite of remaining symptoms $(n=6)$, geographical travel issues to clinic for review $(n=7)$ and other commitments $(n=8)$, with the rest non-contactable for reason of non-return $(n=26)$ despite efforts to do so by email and telephone (figure 1).

Table 2 Changes in HAGOS scores pre-rehabilitation and post-rehabilitation $(n=150)(p<0.001)$

\begin{tabular}{|c|c|c|c|c|c|c|c|}
\hline \multirow[b]{2}{*}{ HAGOS } & \multirow[b]{2}{*}{ Initial mean (SD) } & \multirow[b]{2}{*}{ Discharge mean (SD) } & \multirow[b]{2}{*}{ Mean change (SD) } & \multirow{2}{*}{$\begin{array}{c}\text { Std error mean } \\
\text { change }\end{array}$} & \multicolumn{3}{|c|}{$95 \% \mathrm{Cl}$} \\
\hline & & & & & Lower & Upper & Effect size \\
\hline Pain & $72.9(+/-14.7)$ & $88(+/-11.9)$ & $15.1(+/-15.7)$ & 1.29 & 12.55 & 17.63 & 0.96 \\
\hline Symptoms & $60.3(+/-17.4)$ & $89.1(+/-10.2)$ & $28.8(+/-17.2)$ & 1.40 & 26.10 & 31.64 & 1.68 \\
\hline$A D L$ & $73.9(+/-18.9)$ & $93.2(+/-10.9)$ & $19.2(+/-18.6)$ & 1.52 & 16.23 & 22.24 & 1.03 \\
\hline Sports & $50.7(+/-16.7)$ & $86.3(+/-13.7)$ & $35.6(+/-20)$ & 1.63 & 32.38 & 38.81 & 1.78 \\
\hline PA & $42(+1-38)$ & $66.3(+/-31.4)$ & $24.3(+/-41.4)$ & 3.38 & 17.57 & 30.93 & 0.59 \\
\hline QOL & $36.5(+/-14.7)$ & $66(+/-21.2)$ & $29.5(+/-21.6)$ & 1.77 & 26.04 & 33.03 & 1.36 \\
\hline
\end{tabular}

ADL - activities of daily living; HAGOS - Hip and Groin Outcome Score; PA - participation in physical activity; QOL - quality of living; Sports - sport and recreational activities. 
Table 4 Kinematic changes in cutting after rehabilitation $(n=112)$

\begin{tabular}{|c|c|c|c|c|c|c|c|}
\hline Variable & Start & End & Initial mean (SD) & Discharge mean & Direction & Finding & Effect size \\
\hline Thorax Angles Frontal $\left({ }^{\circ}\right)$ & 0 & 100 & $-0.91(5.7)$ & $6.06(6.6)$ & Contralateral side flexion & Post>Pre & -0.79 \\
\hline Pelvis Angles Transverse $\left(^{\circ}\right)$ & 0 & 100 & $-0.135(4.4)$ & $6.19(4.6)$ & Contralateral rotation & Post>Pre & -0.76 \\
\hline Pelvis Angles Frontal $\left({ }^{\circ}\right)$ & 0 & 100 & $16.05(1.8)$ & $19.48(1.4)$ & Contralateral side flexion & Post>Pre & -0.62 \\
\hline Ankle Angles Sagittal $\left({ }^{\circ}\right)$ & 9 & 75 & $17.39(8.2)$ & $22.11(8.1)$ & Dorsiflexion & Post>Pre & -0.58 \\
\hline Thorax on Pelvis Angles Frontal $\left({ }^{\circ}\right)$ & 16 & 100 & $16.46(4.3)$ & $12.34(5.2)$ & Ipsilateral side flexion & Pre $>$ Post & 0.56 \\
\hline Thorax Angles Transverse $\left({ }^{\circ}\right)$ & 0 & 100 & $-6.02(8.2)$ & $-1.25(8.8)$ & Ipsilateral rotation & Post>Pre & -0.54 \\
\hline Hip Angles Sagittal $\left({ }^{\circ}\right)$ & 0 & 100 & $42.03(14.5)$ & $36.59(14.7)$ & Flexion & Pre $>$ Post & 0.51 \\
\hline Thorax on Pelvis Angles Transverse $\left(^{\circ}\right)$ & 0 & 90 & $5.51(4.0)$ & $8.23(4)$ & Ipsilateral rotation & Post>Pre & -0.46 \\
\hline Hip Angles Frontal( $\left(^{\circ}\right)$ & 67 & 100 & $-19.91(2.1)$ & $-17.64(1.8)$ & Abduction & Pre $>$ Post & -0.36 \\
\hline Knee Angles Sagittal $\left({ }^{\circ}\right)$ & 57 & 100 & $50.85(11.4)$ & $47.76(11.3)$ & Flexion & Pre>Post & 0.33 \\
\hline COM to COP Sagittal (mm) & 4 & 41 & $507(12)$ & $527(16)$ & Anterior & Post>Pre & -0.36 \\
\hline COM to COP Frontal (mm) & 0 & 95 & $-58(101)$ & $-108(10)$ & Contralateral & Post>Pre & 0.40 \\
\hline Ground Contact Time (seconds) & & & $0.38(0.06)$ & $0.36(0.06)$ & & Pre $>$ Post & 0.30 \\
\hline
\end{tabular}

Start-\% point in stance phase when difference started to occur; End—\% point in stance phase when difference ended.

$\mathrm{COM}$, centre of mass; COP, centre of pressure.

\section{HAGOS questionnaire}

A significant improvement across all subsections of HAGOS was seen post intervention (table 2).

\section{Squeeze test}

A significant improvement in squeeze tests was demonstrated at all three angles, with $94 \%(105 / 112)$ of patients achieving painfree squeeze at $0^{\circ}, 45^{\circ}$ and $90^{\circ}$ on RTP (table 3 ).

\section{RTP rates and times}

The pain-free RTP rate of those who entered the study was $73 \%$ $(150 / 205)$, with patients attending $5.1( \pm 1.5)$ appointments prior to RTP. There was no difference in time to RTP based on anatomical diagnosis $(p=0.56)$, and there was no correlation between duration of symptoms prior to rehabilitation and time to RTP $(\mathrm{p}=0.17)$.

\section{Changes in 3D biomechanical analysis of cutting}

Biomechanical analysis of a $110^{\circ}$ cutting manoeuvre $(n=112)$ using the previously described cluster analysis ${ }^{23}$ during initial assessment placed $43 \%$ in cluster $1,21 \%$ in cluster $2 \%$ and $36 \%$ in cluster 3, with no significant difference in RTP times between the clusters $(\mathrm{p}=0.57)$.

Comparative analysis displayed significant changes in postrehabilitation kinetic and kinematic variables (figure 7). The strongest effect size was for increased pelvic rotation towards the direction of intended travel, and greater trunk side flexion towards the direction of intended travel throughout stance phase (table 4). There were medium effect sizes for changes in pelvic tilt towards the direction of intended travel, reduced trunk-on-pelvis side flexion towards the stance leg, reduced hip flexion and increased ankle dorsiflexion. There were small effect sizes for a number of variables, including increased

\section{SAGITTAL}

\section{FRONTAL / TRANSVERSE}

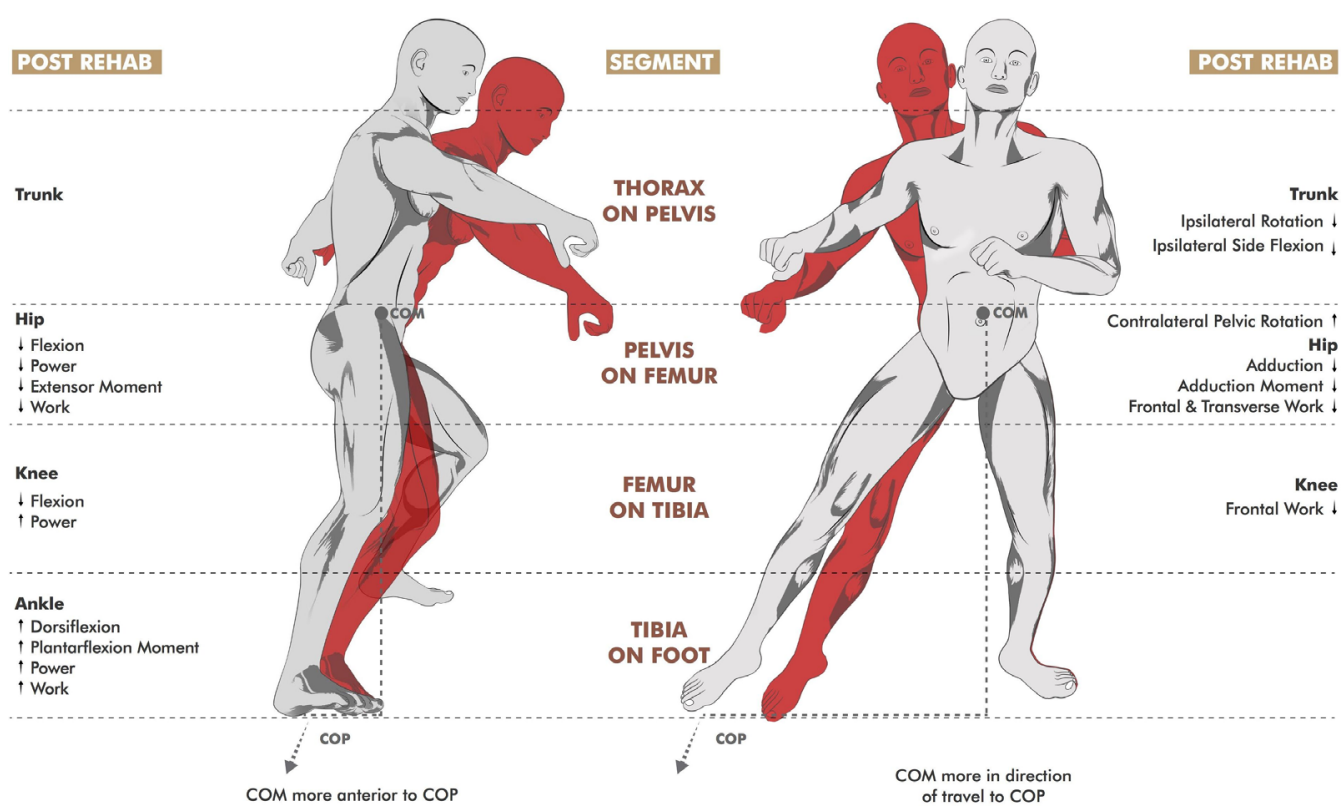

Figure 7 Biomechanical changes in cutting mechanics after rehabilitation (grey figure). COM, centre of mass; COP, centre of pressure. 
Table 5 Kinetic changes in cutting after rehabilitation

\begin{tabular}{|c|c|c|c|c|c|c|c|c|c|}
\hline Variable & Start & End & Initial mean & SD & Discharge mean & SD & Direction & Finding & Effect size \\
\hline Ankle moment sagittal & 6 & 71 & 19.62 & 8.11 & 22.60 & 8.29 & Plantar & Post $>$ Pre & -0.48 \\
\hline Ankle power sagittal & 1 & 24 & -0.03 & 0.03 & -0.05 & 0.03 & Eccentric & Post $>$ Pre & 0.46 \\
\hline Ankle power sagittal & 57 & 83 & 0.05 & 0.04 & 0.07 & 0.04 & Concentric & Post $>$ Pre & -0.46 \\
\hline Hip power sagittal & 68 & 87 & 0.04 & 0.02 & 0.02 & 0.02 & Concentric & Pre $>$ Post & 0.43 \\
\hline Hip moment sagittal & 50 & 89 & 15.31 & 9.95 & 10.57 & 9.98 & Extensor & Pre $>$ Post & 0.41 \\
\hline Knee power sagittal & 43 & 58 & 0.00 & 0.02 & 0.02 & 0.02 & Concentric & Post $>$ Pre & -0.4 \\
\hline Hip moment frontal & 78 & 95 & -7.75 & 0.95 & -5.48 & 0.68 & Adductor & Pre $>$ Post & -0.39 \\
\hline
\end{tabular}

Power units—watts; moment units—Newton-metres/kg; Start—\% point in stance phase when difference started to occur; End-\% point in stance phase when difference ended.

thoracic rotation in the direction of intended travel, reduced hip abduction and knee flexion angle. The centre of mass was more anterior relative to the centre of pressure during the eccentric phase of cutting, more towards the direction of intended travel throughout stance phase after rehabilitation and ground contact time was reduced. There was no difference in the centre of mass velocity (approach speed) at initial contact before $(2.14 \mathrm{~m} / \mathrm{s}( \pm 0.3))$ and after rehabilitation $(2.16$ $\mathrm{m} / \mathrm{s}( \pm 0.29))(\mathrm{p}=0.434)$.

Kinetic analysis demonstrated increased ankle plantar flexion moment post rehabilitation and reduced hip extensor moment as well as reduced hip adduction moment during push-off (table 5). Analysis of total work done at each joint showed a large increase in total work done at the ankle, a moderate reduction in the total work done at the hip and a small reduction at the knee after rehabilitation. This primarily was affected by large increases in work in the sagittal plane of the ankle and moderate reductions in work in all three planes at the hip and frontal plane at the knee (table 6).

\section{DISCUSSION}

We describe a rehabilitation programme for patients with AGP, regardless of clinical entity. The programme was designed to target intersegmental control (across strength, linear and multidirectional drills) and optimise the biomechanics of maximal effort change of direction cutting. The post rehabilitation cohort demonstrated significantly different change of direction strategies during the cutting manoeuvre.

\section{Clinical outcomes (HAGOS, RTP, squeeze test)}

HAGOS subscores for pain, symptoms, activities of daily living and sports/recreation all returned to normative levels ${ }^{36}$ but remained reduced compared with athletes who have never had groin pain. ${ }^{29}$ This pattern of recovery has been identified in similar cohorts previously. ${ }^{37}$ Quality of life and participation in physical activities scores remained below this normal distribution on discharge (table 2). Recovery of quality of life scores have been shown to be inversely associated with longer duration of symptoms. ${ }^{37}$ These lower scores may reflect ongoing self-driven or coach-driven load management and psychological factors relating to the long duration of symptoms, which may continue to improve over time as the patient makes a successful return to sport.

This study examined both HAGOS and pain provocation tests with reported pain-free RTP to improve outcome validity. The demonstrated pain-free RTP rates $(73 \%)$ and times $(9.9 \pm 3.4$ weeks) compare favourably with anatomically specific rehabilitation protocols used by Hölmich (68\% RTP, 18.5 weeks) ${ }^{11}$ and Weir (48\% RTP, 17.3 weeks), ${ }^{10}$ as well as surgical protocols for adductor (63\%-76\% RTP, 14-18 weeks) ${ }^{38-40}$ and pubic pathology (100\% RTP, 13-28 weeks). ${ }^{41} 42$ Both rehabilitation papers are randomised control trials, ${ }^{10} 11$ not prospective case series as in this case; a higher level of evidence ensuring their outcomes did not occur by chance. Of those who made a pain-free RTP, seven patients demonstrated residual symptoms on squeeze test at discharge, suggesting a clinical lag between pain-free RTP and pain-free squeeze at $0^{\circ}, 45^{\circ}$ and $90^{\circ}$. As the median duration of symptoms prior to rehabilitation was over 8 months, the authors consider the mean time to RTP of 9.9 weeks encouraging and believe the intervention may have influenced outcomes.

\section{Biomechanical changes in cutting}

Following rehabilitation, patients demonstrated changes in various biomechanical variables post rehabilitation, which have been shown to be related to cutting performance. These

Table 6 Changes in joint work during cutting after rehabilitation

\begin{tabular}{|c|c|c|c|c|c|c|}
\hline Variable & Initial mean, \% & SD & Discharge mean, \% & SD & Finding & Effect size \\
\hline Total ankle work & 29.57 & 6.89 & 34.84 & 7.68 & Post $>$ Pre & -0.68 \\
\hline Total knee work & 40.43 & 6.40 & 38.88 & 5.55 & Pre $>$ Post & 0.26 \\
\hline Total hip work & 30.00 & 7.66 & 26.28 & 7.25 & Pre $>$ Post & 0.48 \\
\hline Ankle work sagittal & 26.10 & 6.65 & 31.38 & 7.45 & Post $>$ Pre & -0.70 \\
\hline Knee work frontal & 7.89 & 3.18 & 6.79 & 2.89 & Pre $>$ Post & 0.36 \\
\hline Hip work sagittal & 22.43 & 7.10 & 19.64 & 6.31 & Pre $>$ Post & 0.41 \\
\hline Hip work frontal & 4.01 & 1.82 & 3.57 & 1.90 & Pre $>$ Post & 0.24 \\
\hline Hip work transverse & 3.56 & 1.59 & 3.07 & 1.45 & Pre $>$ Post & 0.32 \\
\hline
\end{tabular}

$\%$, percentage of total work done at lower limb. 
include reduced ground contact time, ${ }^{214} 44$ increased centre of mass distance to the centre of pressure in the frontal plane, ${ }^{20}$ reduced trunk side flexion towards the stance leg, ${ }^{21} 43$ reduced knee flexion, ${ }^{44}$ and increased ankle power and plantar flexion moment. ${ }^{21}$ These changes also concurrently reduced the load around the hip and groin as work in all three planes of the hip and the adductor moment were reduced.

\section{Components of rehabilitation}

Exercise selection was based on individual patient competency and progressed according to improvements in segmental control. Without appropriate execution of any exercise, the patient may not achieve the desired training effect or change in segmental control, leading to delayed recovery and commonly an aggravation of symptoms despite appropriate dosage. This approach ensured the programme was set at a level specific to the patient and progressed as quickly or slowly as the individual competency and symptom levels allowed, ensuring the most appropriate exercise selection and recovery time.

Level 1 focused on intersegmental control and strength. Reduced gluteal and iliopsoas activation during hip extension has been suggested to increase anterior hip joint forces, and thus restoring function in both is essential to optimising load distribution in the region. ${ }^{45}$ Loss of lumbopelvic control into anterior pelvic tilt has been shown to increase dynamic femoroacetabular impingement and load across the symphysis pubis, ${ }^{4647}$ and changes in pelvic position relative to the femur have been shown to influence hip muscle action and joint loading at the hip. ${ }^{48} 49$ Hip strength and rate of force development relative to body weight have been shown to correlate positively with cutting performance and biomechanics. ${ }^{50} 51$ Level 1 included exercises that focused on control between individual segments and combined them with compound movements such as deadlift, squat and lunge, which targeted multisegmental coordination while developing strength. It is noteworthy that this study demonstrated a significant improvement in adductor strength in the absence of any isolated adductor strengthening during rehabilitation, perhaps demonstrating the efficacy of restoring pain-free function to the area in improving adductor function.

\section{Linear and multidirectional mechanics}

Acceleration, sprinting and change of direction are the most commonly reported aggravating activities for AGP; therefore, early incorporation of rate of force development and running mechanics, through linear and multidirectional drills, was deemed important. ${ }^{52}$ The linear running drills addressed overstride and dynamic anterior pelvic tilt. Overstride may increase anterior hip joint forces at end range extension ${ }^{54}$ causing increased hip joint load, ${ }^{55}$ while anterior pelvic tilt may increase femoroacetabular contact and pubic symphysis stress. ${ }^{46}$ The linear running programme A and B gradually increased patients' load tolerance and exposure to avoid injury associated with acute spikes in training load on resumption of playing. ${ }^{53}$ Change of direction drills have been shown to be more effective at improving change of direction performance than strength training or sprint training alone. ${ }^{56}$ Progression to the multidirectional drills focused on the rate of force development across all three planes and reactive agility to prepare the patient for sports-specific movements with the associated enhancement in cutting mechanics and performance outlined above.

\section{Limitations}

This was not a randomised trial and there is no explicit control group. The results are impacted by a loss to follow-up rate of $27 \%$ and additionally a further $14 \%$ returning to play without final 3D comparison. An omission was made in the registration of the study in the non-inclusion of an additional secondary outcome measure of RTP.

Future studies should look at the relationship between changes in specific biomechanical variables and outcome measures such as HAGOS and RTP to allow for more targeted rehabilitation strategies, as well as compare the current rehabilitation approach with previously published anatomical specific protocols.

\section{CONCLUSION}

This study demonstrated that a rehabilitation programme focused on intersegmental control improved patients' HAGOS scores and pain provocation tests, and allowed $73 \%$ of patients to return to play pain-free faster than in previous trials. In this study, successful rehabilitation effected a change in cutting biomechanics, which reduced mechanical work across the hip as well as variables associated with improved cutting performance. An approach to rehabilitation based on intersegmental control may offer an alternative to strategies based on specific anatomical diagnoses, and warrants further comparison in randomised control trials.

\section{What are the findings?}

A rehabilitation programme focused on intersegmental control was associated with improvement in a range of outcome measures (return to play, Hip and Groin Outcome Score, squeeze test) in patients with diverse (and multiple) anatomical diagnoses for athletic groin pain.

- Rehabilitation focusing on intersegmental control elicited changes in the change of direction biomechanics associated with improved cutting performance.

- Three-dimensional (3D) biomechanical examination can provide new insights into understanding rehabilitation interventions.

\section{How might it impact on clinical practice in the future?}

Focus attention towards identifying movement strategies as a risk factor for athletic groin pain.

- Allow the focus of rehabilitation to be on intersegmental coordination control rather than specific anatomical structures.

- Identify specific targets for rehabilitation through 3D motion analysis.

Contributors EK was involved in the creation of study design and hypothesis, data collection, carrying out rehabilitation intervention, data analysis, and drafting and revising submission. AFM was involved in the creation of study design and hypothesis, data collection, and drafting and revising submission. CR was involved in the creation of study design, data collection, data analysis and drafting submission. EOR was involved in the creation of study design, data collection and carrying out rehabilitation intervention, and revision of submission. MD was involved in study design, data collection, data analysis and revision of submission. KM was involved in study design, data interpretation and revision of submission. SS was involved in study design, data interpretation and revision of submission. EF was involved in the creation of study design and hypothesis, data collection, and drafting and revising submission.

Funding This research received no specific grant from any funding agency in the public, commercial or not-for-profit sectors. 
Competing interests Sports Surgery Clinic is a private orthopaedic and sports medicine clinic and operates an athletic groin pain programme.

Patient consent Obtained.

Ethics approval The Sports Surgery Clinic Hospital Ethics Committee approved the study (Ref 25EF011), which was registered at ClinicalTrials.gov (NCT02437942)

Provenance and peer review Not commissioned; externally peer reviewed.

Open access This is an open access article distributed in accordance with the Creative Commons Attribution Non Commercial (CC BY-NC 4.0) license, which permits others to distribute, remix, adapt, build upon this work non-commercially, and license their derivative works on different terms, provided the original work is properly cited and the use is non-commercial. See: http://creativecommons.org/ licenses/by-nc/4.0/

(c) Article author(s) (or their employer(s) unless otherwise stated in the text of the article) 2018. All rights reserved. No commercial use is permitted unless otherwise expressly granted.

\section{REFERENCES}

1 Falvey ÉC, King E, Kinsella S, et al. Athletic groin pain (part 1): a prospective anatomical diagnosis of 382 patients--clinical findings, MRI findings and patientreported outcome measures at baseline. Br I Sports Med 2016;50:423-30.

2 Hölmich P. Long-standing groin pain in sportspeople falls into three primary patterns, a "clinical entity" approach: a prospective study of 207 patients. Br J Sports Med 2007:41-247-52.

3 Verrall GM, Henry L, Fazzalari NL, et al. Bone biopsy of the parasymphyseal pubic bone region in athletes with chronic groin injury demonstrates new woven bone formation consistent with a diagnosis of pubic bone stress injury. Am I Sports Med 2008:36:2425-31.

4 Falvey EC, Franklyn-Miller A, McCrory PR. The groin triangle: a patho-anatomical approach to the diagnosis of chronic groin pain in athletes. Br J Sports Med 2009;43:213-20.

5 Serner A, van Eijck $\mathrm{CH}$, Beumer BR, et al. Study quality on groin injury management remains low: a systematic review on treatment of groin pain in athletes. Br J Sports Med 2015;49:813.

6 Weir A, Brukner P, Delahunt E, et al. Doha agreement meeting on terminology and definitions in groin pain in athletes. Br J Sports Med 2015;49:768-74.

7 King E, Ward J, Small L, et al. Athletic groin pain: a systematic review and metaanalysis of surgical versus physical therapy rehabilitation outcomes. $\mathrm{Br}$ I Sports Med 2015:49:1447-51.

8 Machotka Z, Kumar S, Perraton LG. A systematic review of the literature on the effectiveness of exercise therapy for groin pain in athletes. Sports Med Arthrosc Rehabil Ther Technol 2009;1:5.

9 Jansen JA, Mens JM, BackX FJ, et al. Treatment of longstanding groin pain in athletes: a systematic review. Scand J Med Sci Sports 2008;18:263-74.

10 Weir A, Jansen JA, van de Port IG, et al. Manual or exercise therapy for longstanding adductor-related groin pain: a randomised controlled clinical trial. Man Ther 2011;16:148-54.

11 Hölmich P, Uhrskou P, Ulnits L, et al. Effectiveness of active physical training as treatment for long-standing adductor-related groin pain in athletes: randomised trial. Lancet 1999:353:439-43.

12 Hölmich P, Nyvold P, Larsen K. Continued significant effect of physical training as treatment for overuse injury: 8- to 12-year outcome of a randomized clinical trial. Am Sports Med 2011:39:2447-51.

13 Thorborg K, Bandholm T, Hölmich P. Hip- and knee-strength assessments using a hand-held dynamometer with external belt-fixation are inter-tester reliable. Knee Surg Sports Traumatol Arthrosc 2013;21:550-5.

14 Thorborg K, Serner A, Petersen J, et al. Hip adduction and abduction strength profiles in elite soccer players: implications for clinical evaluation of hip adductor muscle recovery after injury. Am J Sports Med 2011:39:121-6.

15 Kloskowska P, Morrissey D, Small C, et al. Movement patterns and muscular function before and after onset of sports-related groin pain: a systematic review with metaanalysis. Sports Med 2016;46:1847-67.

16 Bradshaw CJ, Bundy M, Falvey E. The diagnosis of longstanding groin pain: a prospective clinical cohort study. Br J Sports Med 2008;42:551-4.

17 Brukner PK K. Clinical Sports Medicine. 4th edn. McGraw-Hill: Australia Pty Ltd, 2009

18 Lovell G. The diagnosis of chronic groin pain in athletes: a review of 189 cases. Aust J Sci Med Sport 1995;27:76-9.

19 Havens KL, Sigward SM. Joint and segmental mechanics differ between cutting maneuvers in skilled athletes. Gait Posture 2015;41:33-8.

20 Havens KL, Sigward SM. Whole body mechanics differ among running and cutting maneuvers in skilled athletes. Gait Posture 2015;42:240-5.

21 Marshall BM, Franklyn-Miller AD, King EA, et al. Biomechanical factors associated with time to complete a change of direction cutting maneuver. J Strength Cond Res 2014:28:2845-51
22 Kristianslund E, Krosshaug T. Comparison of drop jumps and sport-specific sidestep cutting: implications for anterior cruciate ligament injury risk screening. Am J Sports Med 2013;41:684-8.

23 Franklyn-Miller A, Richter C, King E, et al. Athletic groin pain (part 2): a prospective cohort study on the biomechanical evaluation of change of direction identifies three clusters of movement patterns. Br J Sports Med 2017:51:460-8.

24 Orchard JW. Men at higher risk of groin injuries in elite team sports: a systematic review. Br J Sports Med 2015:49:798-802.

25 Ryan J, DeBurca N, Mc Creesh K. Risk factors for groin/hip injuries in field-based sports: a systematic review. Br I Sports Med 2014;48:1089-96.

26 Whittaker JL, Small C, Maffey L, et al. Risk factors for groin injury in sport: an updated systematic review. Br J Sports Med 2015:49:803-9.

27 von Elm E, Altman DG, Egger M, et al. The Strengthening the Reporting of Observational Studies in Epidemiology (STROBE) statement: guidelines for reporting observational studies. Lancet 2007;370:1453-7.

28 Li KC, Higgs J, Aisen AM, et al. MRI in osteoarthritis of the hip: gradations of severity. Magn Reson Imaging 1988;6:229-36.

29 Thorborg K, Hölmich P, Christensen R, et al. The Copenhagen Hip and Groin Outcome Score (HAGOS): development and validation according to the COSMIN checklist. Br J Sports Med 2011;45:478-91.

30 Delahunt E, McEntee BL, Kennelly C, et al. Intrarater reliability of the adductor squeeze test in gaelic games athletes. J Athl Train 2011;46:241-5.

31 Delahunt E, Thorborg K, Khan KM, et al. Minimum reporting standards for clinical research on groin pain in athletes. Br J Sports Med 2015;49:775-81.

32 Marshall B, Franklyn-Miller A, Moran K, et al. Biomechanical symmetry in elite rugby union players during dynamic tasks: an investigation using discrete and continuous data analysis techniques. BMC Sports Sci Med Rehabil 2015;7:13.

33 Hoffmann TC, Glasziou PP, Boutron I, et al. Better reporting of interventions: template for intervention description and replication (TIDieR) checklist and guide. BMJ 2014;348:g1687.

34 Pataky TC, Vanrenterghem J, Robinson MA. Zero- vs. one-dimensional, parametric vs. non-parametric, and confidence interval vs. hypothesis testing procedures in onedimensional biomechanical trajectory analysis. J Biomech 2015;48:1277-85.

35 Cohen J. Statistical power analysis for the behavioral sciences. Routledge: Elsevier, 1988.

36 Thorborg K, Branci S, Stensbirk F, et al. Copenhagen hip and groin outcome score (HAGOS) in male soccer: reference values for hip and groin injury-free players. $\mathrm{Br} J$ Sports Med 2014;48:557-9.

37 Thorborg K, Rathleff MS, Petersen $\mathrm{P}$, et al. Prevalence and severity of hip and groin pain in sub-elite male football: a cross-sectional cohort study of 695 players. Scand I Med Sci Sports 2017;27:107-14.

38 Akermark C, Johansson C. Tenotomy of the adductor longus tendon in the treatment of chronic groin pain in athletes. Am J Sports Med 1992;20:640-3.

39 Maffulli N, Loppini M, Longo UG, et al. Bilateral mini-invasive adductor tenotomy for the management of chronic unilateral adductor longus tendinopathy in athletes. $\mathrm{Am} J$ Sports Med 2012;40:1880-6.

40 Robertson IJ, Curran C, McCaffrey N, et al. Adductor tenotomy in the management of groin pain in athletes. Int J Sports Med 2011:32:45-8.

41 Hechtman KS, Zvijac JE, Popkin CA, et al. A minimally disruptive surgical technique for the treatment of osteitis pubis in athletes. Sports Health 2010:2:211-5.

42 Williams PR, Thomas DP, Downes EM. Osteitis pubis and instability of the pubic symphysis. When nonoperative measures fail. Am I Sports Med 2000;28:350-5.

43 Sasaki S, Nagano Y, Kaneko $S$, et al. The relationship between performance and trunk movement during change of direction. J Sports Sci Med 2011;10:112-8.

44 Dai B, Garrett WE, Gross MT, et al. The effects of 2 landing techniques on knee kinematics, kinetics, and performance during stop-jump and side-cutting tasks. Am J Sports Med 2015:43:466-74.

45 Lewis CL, Sahrmann SA, Moran DW. Anterior hip joint force increases with hip extension, decreased gluteal force, or decreased iliopsoas force. J Biomech 2007:40:3725-31.

46 Birmingham PM, Kelly BT, Jacobs $R$, et al. The effect of dynamic femoroacetabular impingement on pubic symphysis motion: a cadaveric study. Am I Sports Med 2012:40:1113-8.

47 Ross JR, Nepple JJ, Philippon MJ, et al. Effect of changes in pelvic tilt on range of motion to impingement and radiographic parameters of acetabular morphologic characteristics. Am I Sports Med 2014;42:2402-9.

48 Dostal WF, Soderberg GL, Andrews JG. Actions of hip muscles. Phys Ther 1986:66:351-9.

49 Bergmann G, Graichen F, Rohlmann A. Hip joint contact forces during stumbling. Langenbecks Arch Surg 2004;389:53-9.

50 Spiteri T, Cochrane JL, Hart NH, et al. Effect of strength on plant foot kinetics and kinematics during a change of direction task. Eur I Sport Sci 2013;13:646-52.

51 Delaney JA, Scott TJ, Ballard DA, et al. Contributing factors to change-ofdirection ability in professional rugby league players. J Strength Cond Res 2015;29:2688-96

52 Bergmann $\mathrm{G}$, Kniggendorf $\mathrm{H}$, Graichen F, et al. Influence of shoes and heel strike on the loading of the hip joint. J Biomech 1995;28:817-27. 


\section{Original article}

53 Blanch P, Gabbett TJ. Has the athlete trained enough to return to play safely? The acute:chronic workload ratio permits clinicians to quantify a player's risk of subsequent injury. Br J Sports Med 2016;50:471-5.

54 Lewis CL, Sahrmann SA, Moran DW. Effect of hip angle on anterior hip joint force during gait. Gait Posture 2010;32:603-7.
55 Bergmann G, Graichen F, Rohlmann A. Hip joint loading during walking and running, measured in two patients. J Biomech 1993;26:969-90.

56 Brughelli M, Cronin J, Levin G, et al. Understanding change of direction ability in sport: a review of resistance training studies. Sports Med 2008;38:1045-63. 